\title{
Distribution of Deviation Distance to Alternative Fuel Stations*
}

\author{
Masashi Miyagawa \\ Department of Regional Social Management, University of Yamanashi, Kofu, Japan \\ Email: mmiyagawa@yamanashi.ac.jp
}

Received March 8, 2013; revised April 10, 2013; accepted April 25, 2013

Copyright (C) 2013 Masashi Miyagawa. This is an open access article distributed under the Creative Commons Attribution License, which permits unrestricted use, distribution, and reproduction in any medium, provided the original work is properly cited.

\begin{abstract}
This paper derives the distribution of the deviation distance to visit an alternative fuel station. Distance is measured as the Euclidean distance on a continuous plane. The distribution explicitly considers the vehicle range and whether the round trip between origin and destination can be made. Three cases are examined: fuel is available at both origin and destination, fuel is available at either origin or destination, and fuel is available at neither origin nor destination. The analytical expressions for the distribution demonstrate how the vehicle range, the shortest distance, and the refueling availability at origin and destination affect the deviation distance. The distribution will thus be useful to estimate the number of vehicles refueled at a station.
\end{abstract}

Keywords: Location; Flow Demand; Vehicle Range; Shortest Distance; Round Trip

\section{Introduction}

Alternative fuel vehicles powered by electricity, hydrogen, and biofuels have been promoted because of environmental, geopolitical, and financial concerns. The transition from gasoline engine vehicles to alternative fuel vehicles, however, would be difficult. Major barriers to the transition are the scarcity of refueling stations and the limited range of vehicles.

A sufficient number of alternative fuel stations has been calculated. Melaina [1] developed three estimation approaches based on the number of existing gasoline stations, metropolitan land areas, and lengths of major roads. Melaina and Bremson [2] proposed a sufficient level of station coverage that meets the refueling needs of the general population in urban areas. Nicholas et al. [3] developed a GIS model for siting hydrogen stations and examined the effect of the number of stations on the average driving time to the nearest station. Nicholas and Ogden [4] studied the regional variation in the number of stations needed to achieve a travel time target. Honma and Kurita [5] obtained the optimal number of hydrogen stations that minimizes the sum of operation and transportation costs.

An efficient location of alternative fuel stations has

*This research was supported by Grant-in-Aid for Scientific Research (C) (24510187). also been studied. Kuby and Lim [6] developed the flow refueling location model (FRLM) for optimally locating refueling facilities. The FRLM locates $p$ facilities to maximize the total flow volume that can be refueled. Kuby et al. [7] applied the FRLM to the location of hydrogen stations in Florida. Lim and Kuby [8] presented three heuristic algorithms for the FRLM. Capar and Kuby [9] proposed an efficient formulation of the FRLM that makes it possible to solve large problems. The FRLM was extended by Kuby and Lim [10] to add candidate sites along network arcs, by Upchurch et al. [11] to include the capacity of refueling facilities, and by Kim and Kuby [12] to allow drivers to deviate from their shortest paths.

Refueling stations are typical flow demand facilities in that demand for service can be expressed as flow rather than point. In fact, drivers usually refuel their vehicles on pre-planned paths from origin to destination. Other examples of flow demand facilities include convenience stores, automated teller machines, and nursery schools. Flow demand was first introduced into facility location models by Hodgson [13] who considered the location of facilities that minimizes the total deviation time from work journeys. Hodgson [14] and Berman et al. [15] formulated a location model, which Hodgson called the flow capturing model, for locating facilities on a network so as to maximize the total flow covered. Hodgson et al. 
[16] applied the model to the actual road network in Edmonton, Canada. The model was generalized by Berman et al. [17] to consider the possibility of deviations, by Averbakh and Berman [18] to take account of multicounting, by Zeng et al. [19] to include consumers' preferences, and by Tanaka and Furuta [20] to deal with facilities of different sizes and attractions. Berman [21] introduced four location problems that combine demand coverage problems with flow coverage problems. Zeng et al. [22] proposed a generalized flow-interception location-allocation model for effectively locating facilities on a network with flow-based demand.

In this paper, we derive the distribution of the deviation distance to visit an alternative fuel station. The distribution that shows how the deviation distance is distributed will supply building blocks for location models of refueling stations. For example, the distribution can be used to estimate the number of vehicles refueled at a station, because refueling demand generally decreases with the deviation distance. Given the location of stations and the set of origin-destination pairs, the distribution of the deviation distance can be numerically calculated. The result, however, depends on the specific data and cannot be applied to other situations. For examining fundamental characteristics of the deviation distance, analytical expressions are necessary. Analytical expressions are also useful to interpret and comprehend numerical results.

Although Miyagawa [23] also derived the distribution of the deviation distance, the present paper differs in considering the vehicle range, which is a significant element for alternative fuel vehicles. Even if a station is available within a deviation distance that drivers can tolerate, the vehicle cannot always complete the trip. In fact, if the distance from origin to the station or from the station to destination is greater than the vehicle range, the vehicle cannot complete the trip. We therefore focus on whether the vehicle can make the round trip between origin and destination.

The remainder of this paper is organized as follows. The next section defines the deviation distance and the distribution of the deviation distance. The following sections derive the distribution of the deviation distance for three cases: fuel is available at both origin and destination, fuel is available at either origin or destination, and fuel is available at neither origin nor destination. The final section presents concluding remarks.

\section{Deviation Distance}

Consider trips using alternative fuel vehicles of range $r$. Distance is measured as the Euclidean distance on a continuous plane. Origins and destinations are assumed to be uniformly and independently distributed over the study region. This assumption facilitates the analytical treatment of the distribution of the deviation distance. In fact, the uniformity assumption has frequently been used in continuous transportation models [24-26]. In addition, the uniform demand serves as a basis for further analyses with non-uniform demand. For example, more generalized travel demand can be incorporated by using spatial interaction models [27].

Drivers are assumed to deviate from their shortest paths to refuel their vehicles. Let $t$ and $U$ be the shortest distance between origin and destination and the deviation distance to visit a station, respectively. The deviation distance is defined as the sum of the distances from origin $O$ to the station and from the station to destination $D$. Let $F(u)$ be the volume of flow such that $U \leq u$. We call $F(u)$ the distribution of the deviation distance. The region that a driver can cover within the deviation distance $u$ forms an ellipse, the foci of which are at $O$ and $D$, as shown in Figure 1. Recall that an ellipse is defined as the locus of points such that the sum of the distances to two fixed points (foci) remains constant. If the ellipse contains a station, the station is available within the deviation distance $u$. This also means that the midpoint of the $O-D$ path lies in the same ellipse but centered at the station. Thus, $F(u)$ can be obtained by calculating the volume of flow such that the midpoint of the $O-D$ path lies in the ellipse centered at the station. Not only the deviation distance but also the possibility of making the round trip should be taken into account. This possibility depends on the vehicle range and the refueling availability at origin and destination. In the following sections, we derive the distribution of the deviation distance $F(u)$ for three different cases of the refueling availability at origin and destination.

\section{Fuel Is Available at Both Origin and Destination}

First, we assume that fuel is available at both origin $O$ and destination $D$. Then the vehicle can start at $O$ with full tank of fuel. Note that plug-in electric vehicles can be charged at home or work place. Note also that this assumption also applies to long distance trips where $O$ and $D$ represent other stations.

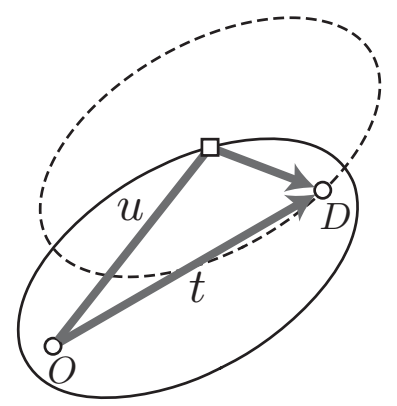

Figure 1. Deviation distance to a station. 
To refuel at a station and complete the round trip, both $O$ and $D$ must be within the distance $r$ of the station. In fact, the vehicle can reach the station, fill up, go to $D$, fill up again, turn round, fill up again at that same station, and return to $O$. Thus, both $O$ and $D$ must be in the circle $C$ centered at the station with radius $r$, as depicted in Figure 2. This means that the midpoint of the $O-D$ path must be in the shaded region in Figure 2. This region is the intersection of the two circles which are obtained by moving the circle $C$ by $t / 2$ in the direction parallel to the $O-D$ path and in the opposite direction.

For $U \leq u$, the midpoint of the $O-D$ path must be in the ellipse centered at the station, as discussed in the previous section. Set the origin of the coordinate system at the station, as shown in Figure 3. The locus such that $U=u$ is the ellipse expressed as

$$
\frac{4 x^{2}}{u^{2}}+\frac{4 y^{2}}{u^{2}-t^{2}}=1
$$

where $t \leq u \leq 2 r . F(u)$ is obtained by calculating the area of the ellipse in the intersection of the two circles as

$$
\begin{aligned}
F(u)= & \frac{2 \sqrt{u^{2}-t^{2}}}{u} \int_{0}^{\alpha} \sqrt{u^{2}-4 x^{2}} \mathrm{~d} x \\
& +4 \int_{\alpha}^{r-t / 2} \sqrt{r^{2}-\left(x+\frac{t}{2}\right)^{2}} \mathrm{~d} x
\end{aligned}
$$

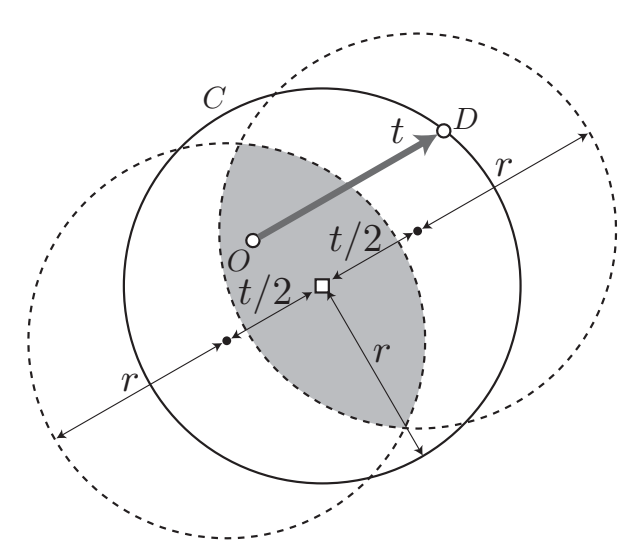

Figure 2. Flow that can be refueled at a station.

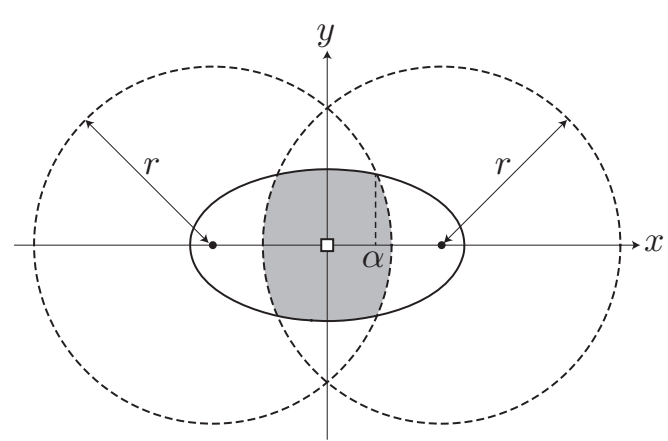

Figure 3. Contour of the deviation distance. where

$$
\alpha=\frac{2 r u-u^{2}}{2 t} .
$$

The distribution of the deviation distance $F(u)$ is shown in Figure 4. The longer the vehicle range $r$ and the shorter the shortest distance $t$, the more the volume of flow that can be refueled within the deviation distance $u$. Note that if $t \leq r$, no vehicle uses the station, because the vehicle can reach $D$ without refueling. Since $t \leq u \leq 2 r, F(2 r)$ represents the total volume of flow that can be refueled at the station.

\section{Fuel Is Available at Either Origin or Destination}

Next, we assume that fuel is available at either origin $O$ or destination $D$. Without loss of generality, we assume that fuel is available at only $O$. If the round trip is considered, the vehicle is required to reach $D$ with at least half a tank remaining.

To refuel at a station and complete the round trip, $O$ must be within the distance $r$ of the station and $D$ must be within the distance $r / 2$ of the station. In fact, the vehicle can reach the station, fill up, go to $D$, turn round, fill up again at that same station, and return to $O$. Thus, $O$ must be in the circle $C_{1}$ centered at the station with radius $r$ and $D$ must be in the circle $C_{2}$ centered at the station with radius $r / 2$, as depicted in Figure 5 . This means that the midpoint of the $O-D$ path must be in the shaded region in Figure 5. This region is the intersection of the two circles which are obtained by moving the circle $C_{1}$ by $t / 2$ in the direction parallel to the $O-D$ path and moving the circle $C_{2}$ by $t / 2$ in the opposite direction.

$F(u)$ is obtained by calculating the area of the ellipse (1) in the intersection of the two circles, as shown in Figure 6. If $t \leq u \leq 2 r-t$,

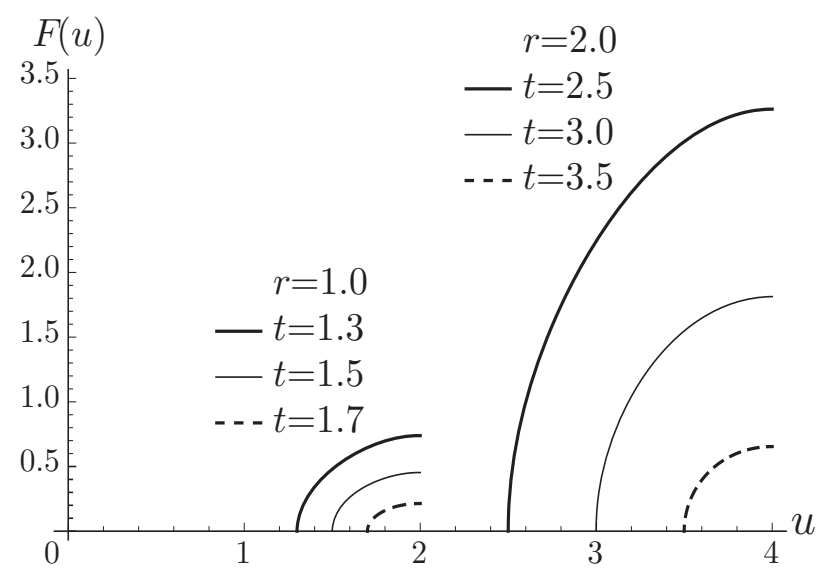

Figure 4. Distribution of the deviation distance. 


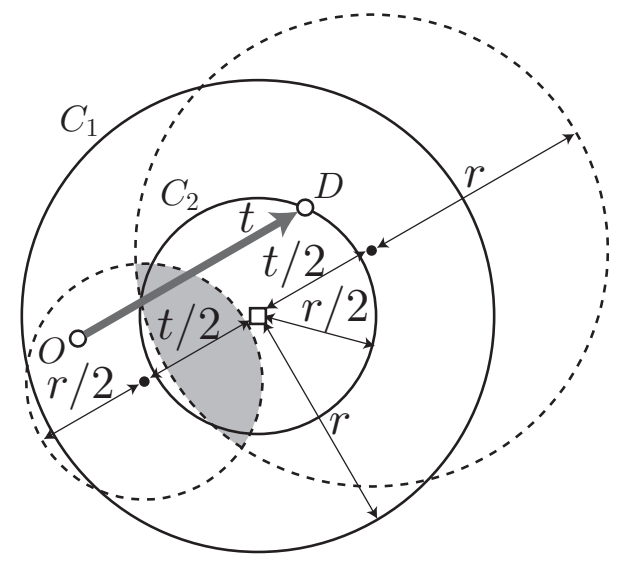

Figure 5. Flow that can be refueled at a station.
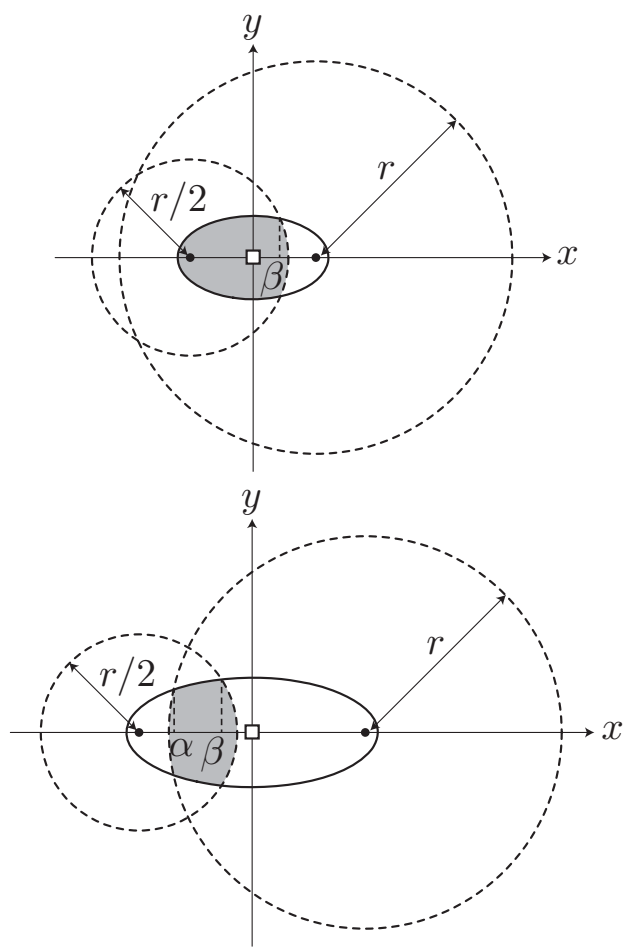

Figure 6. Contour of the deviation distance.

$$
\begin{aligned}
F(u)= & \frac{\sqrt{u^{2}-t^{2}}}{u} \int_{-u / 2}^{\beta} \sqrt{u^{2}-4 x^{2}} \mathrm{~d} x \\
& +2 \int_{\beta}^{r / 2-t / 2} \sqrt{\left(\frac{r}{2}\right)^{2}-\left(x+\frac{t}{2}\right)^{2}} \mathrm{~d} x,
\end{aligned}
$$

and if $2 r-t<u \leq 3 r / 2$,

$$
\begin{aligned}
F(u)= & \frac{\sqrt{u^{2}-t^{2}}}{u} \int_{\alpha}^{\beta} \sqrt{u^{2}-4 x^{2}} \mathrm{~d} x+2 \int_{t / 2-r}^{\alpha} \sqrt{r^{2}-\left(x-\frac{t}{2}\right)^{2}} \mathrm{~d} x \\
& +2 \int_{\beta}^{r / 2-t / 2} \sqrt{\left(\frac{r}{2}\right)^{2}-\left(x+\frac{t}{2}\right)^{2}} \mathrm{~d} x,
\end{aligned}
$$

where

$$
\alpha=\frac{u^{2}-2 r u}{2 t}, \beta=\frac{r u-u^{2}}{2 t} .
$$

The distribution of the deviation distance $F(u)$ is shown in Figure 7. The volume of flow that can be refueled is smaller than that of the case where fuel is available at both origin and destination.

\section{Fuel Is Available at Neither Origin Nor Destination}

Finally, we assume that fuel is available at neither origin $O$ nor destination $D$. We also assume that the vehicle starts at $O$ with half a tank of fuel and reaches $D$ with at least half a tank remaining, as suggested by Kuby and Lim [6]. This assumption ensures that the round trip can be made repeatedly.

To refuel at a station and complete the round trip, both $O$ and $D$ must be within the distance $r / 2$ of the station. In fact, the vehicle can reach the station, fill up, go to $D$, turn round, fill up again at that same station, and return to $O$. Thus, both $O$ and $D$ must be in the circle $C$ centered at the station with radius $r / 2$, as depicted in Figure 8. This means that the midpoint of the $O-D$ path must be in the shaded region in Figure 8. This region is the intersection of the two circles which are obtained by moving the circle $C$ by $t / 2$ in the direction parallel to the $O-D$ path and in the opposite direction.

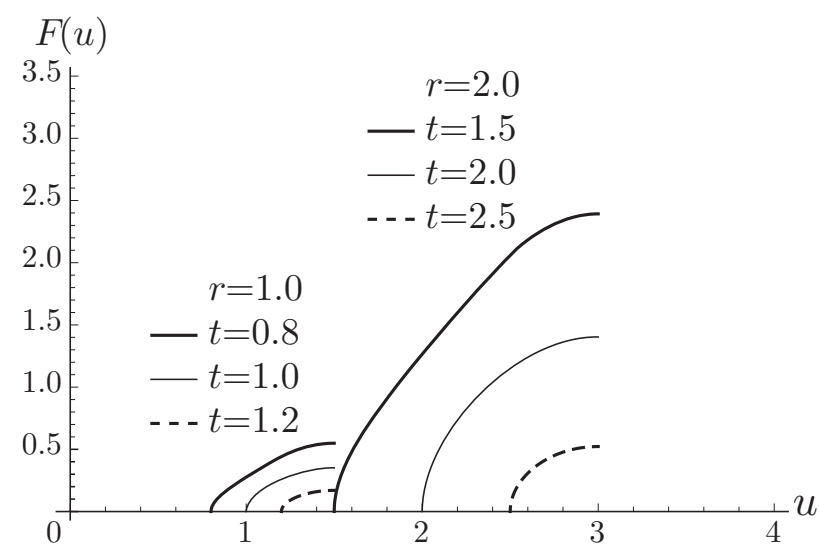

Figure 7. Distribution of the deviation distance.

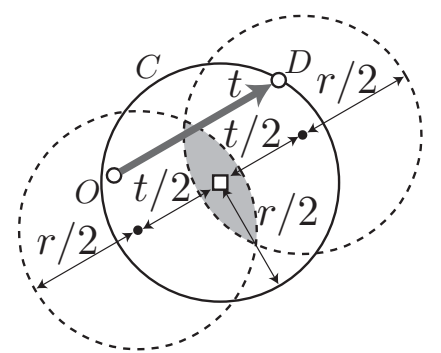

Figure 8. Flow that can be refueled at a station. 
$F(u)$ is obtained by calculating the area of the ellipse (1) in the intersection of the two circles, as shown in Figure 9. If $t \leq u \leq r-t$,

$$
F(u)=\frac{\pi u}{4} \sqrt{u^{2}-t^{2}},
$$

and if $r-t<u \leq r$,

$$
\begin{aligned}
F(u)= & \frac{2 \sqrt{u^{2}-t^{2}}}{u} \int_{0}^{\alpha} \sqrt{u^{2}-4 x^{2}} \mathrm{~d} x \\
& +4 \int_{\alpha}^{r / 2-t / 2} \sqrt{\left(\frac{r}{2}\right)^{2}-\left(x+\frac{t}{2}\right)^{2}} \mathrm{~d} x,
\end{aligned}
$$

where

$$
\alpha=\frac{r u-u^{2}}{2 t} .
$$

The distribution of the deviation distance $F(u)$ is shown in Figure 10. In this case, only short distance trips can visit the station. The total volume of flow that can be refueled is smaller than the other two cases.

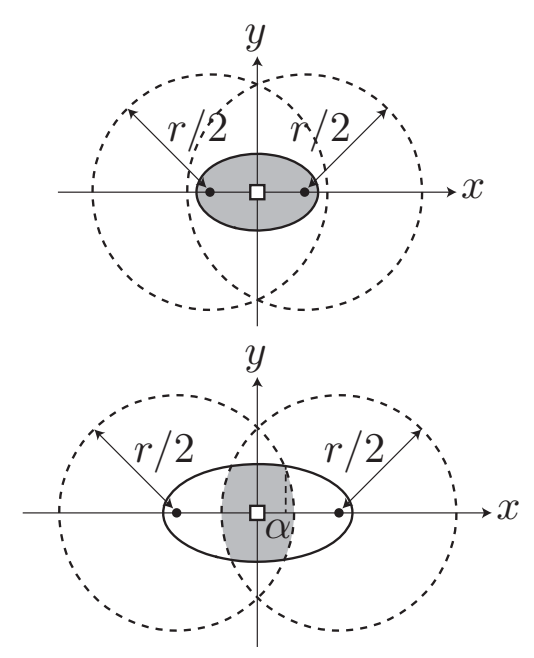

Figure 9. Contour of the deviation distance.

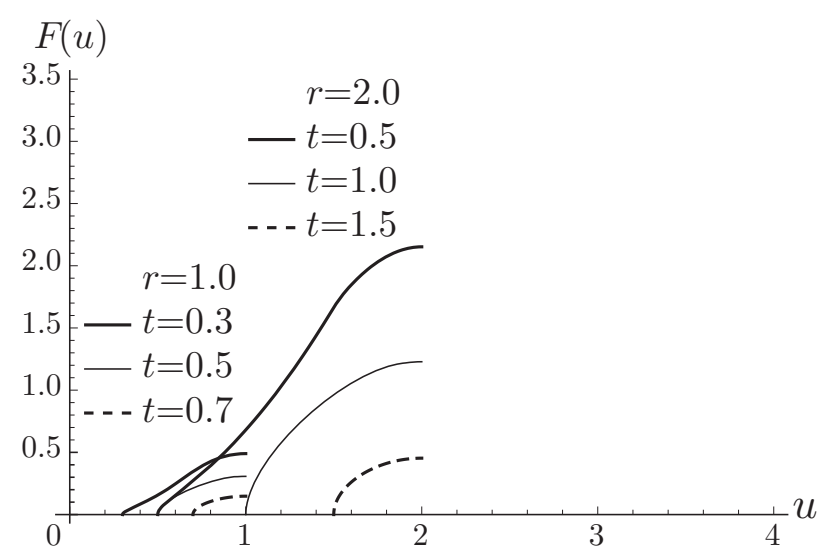

Figure 10. Distribution of the deviation distance.

\section{Conclusions}

This paper has derived the distribution of the deviation distance to visit an alternative fuel station. The major characteristic of the paper is that the focus is on whether the round trip between origin and destination can be made. The distribution that explicitly considers the vehicle range will give a more appropriate framework for the deviation distance of alternative fuel vehicles.

The analytical expressions for the distribution demonstrate how the vehicle range, the shortest distance, and the refueling availability at origin and destination affect the deviation distance. Note that finding these relationships by using discrete network models requires computation of the deviation distance for each combination of the parameters. The relationships are useful to estimate the number of vehicles refueled at a station and sufficient capacity of the station. The sufficient capacity can be used as an input in location models of refueling stations.

In this paper, we implicitly assume the early stages of an alternative fuel industry where stations are sparse. If many stations are available, drivers will choose the best station that minimizes the deviation distance. Incorporating the competition among stations would be a topic for future research.

\section{REFERENCES}

[1] M. W. Melaina, "Initiating Hydrogen Infrastructures: Preliminary Analysis of a Sufficient Number of Initial Hydrogen Stations in the US," International Journal of Hydrogen Energy, Vol. 28, No. 7, 2003, pp. 743-755. doi:10.1016/S0360-3199(02)00240-9

[2] M. Melaina and J. Bremson, "Refueling Availability for Alternative Fuel Vehicle Markets: Sufficient Urban Station Coverage," Energy Policy, Vol. 36, No. 8, 2008, pp. 3233-3241. doi:10.1016/j.enpol.2008.04.025

[3] M. A. Nicholas, S. L. Handy and D. Sperling, "Using Geographic Information Systems to Evaluate Siting and Networks of Hydrogen Stations," Transportation Research Record, Vol. 1880, 2004, pp. 126-134. doi:10.3141/1880-15

[4] M. A. Nicholas and J. Ogden, "Detailed Analysis of Urban Station Siting for California Hydrogen Highway Network," Transportation Research Record, Vol. 1983, 2006, pp. 121-128. doi:10.3141/1983-17

[5] Y. Honma and O. Kurita, "A Mathematical Model on the Optimal Number of Hydrogen Stations with Respect to the Diffusion of Fuel Cell Vehicles," Journal of the Operations Research Society of Japan, Vol. 51, No. 2, 2008, pp. 166-190.

[6] M. Kuby and S. Lim, "The Flow-Refueling Location Problem for Alternative-Fuel Vehicles," Socio-Economic Planning Sciences, Vol. 39, No. 2, 2005, pp. 125-145. doi:10.1016/j.seps.2004.03.001

[7] M. Kuby, L. Lines, R. Schultz, Z. Xie, J. G. Kim and S. Lim, "Optimization of Hydrogen Stations in Florida Us- 
ing the Flow-Refueling Location Model," International Journal of Hydrogen Energy, Vol. 34, No. 15, 2009, pp. 6045-6064. doi:10.1016/j.ijhydene.2009.05.050

[8] S. Lim and M. Kuby, "Heuristic Algorithms for Siting Alternative-Fuel Stations Using the Flow-Refueling Location Model," European Journal of Operational Research, Vol. 204, No. 1, 2010, pp. 51-61. doi:10.1016/i.ejor.2009.09.032

[9] I. Capar and M. Kuby, "An Efficient Formulation of the Flow Refueling Location Model for Alternative-Fuel Stations," IIE Transactions, Vol. 44, No. 8, 2012, pp. 622636. doi:10.1080/0740817X.2011.635175

[10] M. Kuby and S. Lim, "Location of Alternative-Fuel Stations Using the Flow-Refueling Location Model and Dispersion of Candidate Sites on Arcs," Networks and Spatial Economics, Vol. 7, No. 2, 2007, pp. 129-152. doi:10.1007/s11067-006-9003-6

[11] C. Upchurch, M. Kuby and S. Lim, "A Model for Location of Capacitated Alternative-Fuel Stations," Geographical Analysis, Vol. 41, No. 1, 2009, pp. 85-106. doi:10.1111/j.1538-4632.2009.00744.x

[12] J. G. Kim and M. Kuby, "The Deviation-Flow Refueling Location Model for Optimizing a Network of Refueling Stations," International Journal of Hydrogen Energy, Vol. 37, No. , 2012, pp. 5406-5420. doi:10.1016/i.ijhydene.2011.08.108

[13] M. J. Hodgson, "The Location of Public Facilities Intermediate to the Journey to Work," European Journal of Operational Research, Vol. 6, No. 2, 1981, pp. 199-204. doi:10.1016/0377-2217(81)90208-3

[14] M. J. Hodgson, "A Flow-Capturing Location-Allocation Model," Geographical Analysis, Vol. 22, No. 3, 1990, pp. 270-279. doi:10.1111/j.1538-4632.1990.tb00210.x

[15] O. Berman, R. C. Larson and N. Fouska, "Optimal Location of Discretionary Service Facilities," Transportation Science, Vol. 26, No. 3, 1992, pp. 201-211. doi:10.1287/trsc.26.3.201

[16] M. J. Hodgson, K. E. Rosing and A. L. G. Storrier, “Applying the Flow-Capturing Location-Allocation Model to an Authentic Network: Edmonton, Canada," European Journal of Operational Research, Vol. 90, No. 3, 1996, pp. 427-443. doi:10.1016/0377-2217(95)00034-8

[17] O. Berman, D. Bertsimas and R.C. Larson, "Locating
Discretionary Service Facilities, II: Maximizing Market Size, Minimizing Inconvenience," Operations Research, Vol. 43, No. 4, 1995, pp. 623-632. doi:10.1287/opre.43.4.623

[18] I. Averbakh and O. Berman, "Locating Flow-Capturing Units on a Network with Multi-Counting and Diminishing Returns to Scale," European Journal of Operational Research, Vol. 91, No. 3, 1996, pp. 495-506. doi:10.1016/0377-2217(94)00369-6

[19] W. Zeng, M. J. Hodgson and I. Castillo, "The Pickup Problem: Consumers' Locational Preferences in Flow Interception," Geographical Analysis, Vol. 41, No. 1, 2009, pp. 107-126. doi:10.1111/j.1538-4632.2009.00741.x

[20] K. Tanaka and T. Furuta, "A Hierarchical Flow Capturing Location Problem with Demand Attraction Based on Facility Size, and Its Lagrangian Relaxation Solution Method," Geographical Analysis, Vol. 44, No. 1, 2012, pp. 15-28. doi:10.1111/j.1538-4632.2011.00837.x

[21] O. Berman, "Deterministic Flow-Demand Location Problems," Journal of the Operational Research Society, Vol. 48, No. 1, 1997, pp. 75-81.

[22] W. Zeng, I. Castillo and M. J. Hodgson, "A Generalized Model for Locating Facilities on a Network with FlowBased Demand," Networks and Spatial Economics, Vol. 10, No. 4, 2010, pp. 579-611. doi:10.1007/s11067-008-9073-8

[23] M. Miyagawa, "Distributions of Rectilinear Deviation Distance to Visit a Facility," European Journal of Operational Research, Vol. 205, No. 1, 2010, pp. 106-112. doi:10.1016/j.ejor.2009.12.002

[24] S. Chien and P. Schonfeld, "Optimization of Grid Transit System in Heterogeneous Urban Environment," Journal of Transportation Engineering, Vol. 123, No. 1, 1997, pp. 28-35. doi:10.1061/(ASCE)0733-947X(1997)123:1(28)

[25] M. M. Aldaihani, L. Quadrifoglio, M. M. Dessouky and R. Hall, "Network Design for a Grid Hybrid Transit Service," Transportation Research Part A, Vol. 38, No. 7, 2004, pp. 511-530. doi:10.1016/j.tra.2004.05.001

[26] C. F. Daganzo, "Structure of Competitive Transit Networks," Transportation Research Part B, Vol. 44, No. 4, 2010, pp. 434-446. doi:10.1016/j.trb.2009.11.001

[27] J. R. Roy, "Spatial Interaction Modelling," Springer-Verlag, Berlin, 2010. 